\title{
Epidemiology of Congenital Anomalies among the Children born in Different Hospitals under Sylhet Division in Bangladesh- a Retrospective Study
}

\author{
Madhabi Lata Shuma ${ }^{1}$, Shimul Halder ${ }^{2}$ and Bidyut Kanti Datta ${ }^{1}$ \\ ${ }^{1}$ Department of Pharmacy, Stamford University Bangladesh, 51 Siddeswari Road, Ramna, Dhaka-1217, Bangladesh \\ ${ }^{2}$ Department of Pharmaceutical Technology, University of Dhaka, Dhaka-1000, Bangladesh
}

Received: August 03, 2015; Accepted: August 30, 2015; Published (web): January 31, 2016

\begin{abstract}
Congenital anomalies play a significant role in perinatal and neonatal mortality. Frequency of these congenital anomalies varies in different populations. Epidemiology is the study of frequency, distribution and determinants of disease in populations. The mission of epidemiology is to contribute to the understanding of etiology and prevention of disease and to improve the health of public through excellence in research. So, this study was conducted to find out proportion, types and immediate outcome of congenital anomalies. In this retrospective and cross-sectional study all patients admitted with congenital anomalies in different hospitals of Sylhet division in Bangladesh from October 2012 to January 2013 (04 months) were included. At first, the patients were examined for major and minor congenital anomalies and recorded properly. Diagnosis of congenital anomalies was based on ultrasonography and clinical evaluation of the fetus/newborn by experienced neonatologist. Major and minor congenital anomalies were categorized depending on their life threatening effects. Various data which were obtained was analyzed by using SPSS 13. Rates and proportions were calculated with 95\% confidence interval. The proportions were compared using students $\mathrm{T}$-test. Level of significance was set at $\mathrm{P}<0.05$. The most frequently occurring anomalies involved the central nervous system (28.33\%) of which myelomeningocele was the commonest CNS defect (9.09\%). According to data, females were more susceptible to myelomeningocele than the male patients. The second frequently occurring congenital anomaly was associated with cardiovascular system (15\%). Patients with urogenital anomalies $(6.67 \%)$ were male, except for one who had ambiguous genitalia. Congenital anomaly associated with gastrointestinal -, respiratory -, musculoskeletal -, chromosomal - and dysmorphism- were noted as $11.67 \%, 5 \%, 6.67 \%, 5 \%$ and $3.33 \%$, respectively. About $11 \%$ congenital anomalies were found to be linked to other body part system. After analysis of mothers' lifestyle, it was likely to conclude that more stress and efforts should be given on the role of peri-conceptional vitamin supplementation to the pregnant mother. Folic acid was found to be the primary agent for prevention of congenital defects.
\end{abstract}

Key words: Congenital anomaly, myelomeningocele, dysmorphism Clubfoot, hypospadias, cleft lip/palate, newborns encephalocele, Down's syndrome

\section{INTRODUCTION}

A congenital anomaly is a structural abnormality or defect that is present at birth. Such abnormalities commonly are called as "birth defects". A congenital anomaly or birth defect can affect any part of the body and can affect the body part in different ways. Some congenital anomalies affect the way a person

Correspondence to: Shimul Halder

E-mail: shimulpht@du.ac.bd

Dhaka Univ. J. Pharm. Sci. 14(2): 225-230, 2015 (December) looks, while others affect function of part of the body. ${ }^{1-3}$

Major congenital anomalies are defined as conditions that results a malformation, deformation or disruption in one or more parts of body which are present at birth and have a serious adverse effect on health, development or functional ability. The etiology of congenital abnormality may be genetic (30-40\%) or environmental (5-10\%). Among genetic causes, chromosomal abnormality makes up about $6 \%$, single gene disorders about $7.5 \%$ and multi- 
factorial factors (Hereditary and other) about $20 \%$. In about $60 \%$ of cases, the cause is not known. ${ }^{4-6}$ Environmental factors which were found to included are -
$>$ use of drugs
$>$ chemotherapy
$>$ radiation exposure
$>$ cousin marriages
$>$ lack of nutrition

Early intrauterine period (between the 3rd and the 8th week of gestation) is the vital period of life for the normal development of organs. Any insult within that period may result in congenital abnormalities. It can further be argued that interventions within this period targeted at preventing insults (or removing the effects of insults) to the developing fetus will reduce the likelihood of an abnormality developing. Congenital anomalies involving the brain are reported to have the highest incidence at 10/1000 live births compared to heart at $8 / 1000$, kidneys at $4 / 1000$, limb at $1 / 1000$ while all others have a combined incidence of 6/1000 live births. $^{7}$

Congenital anomalies are either single isolated defects or present as multiple anomalies in a single individual. A syndrome is defined as a pattern of multiple abnormalities that are related topathophysiology and is a result of common, defined etiology. Major congenital anomalies occur in approximately $2-3 \%$ of births with a variable frequency in different populations ranging from $1.07 \%$ in Japan to $4.3 \%$ in Taiwan. Congenital anomalies or birth defects are relatively common, affecting $3 \%$ to 5\% of live-births in the United States (USA) and $2.1 \%$ in Europe. These account for $8 \%$ to $15 \%$ of perinatal deaths and $13 \%$ to $16 \%$ of neonatal deaths in India. For more than two decades, congenital anomalies have been the leading cause of infant mortality in the USA. The morbidity and disability experienced by surviving children also has a major public health impact. ${ }^{8,9}$

Congenital abnormality plays a major role in morbidity and mortality of children. However, the treatment and rehabilitation of these children with congenital abnormality is very costly. Hence it is required to identify causative and risk factors and to prevent them early, where possible. The birth of an infant with major malformations whether diagnosed ante-natally or not evokes an emotional parental response. Early recognition of anomalies is important for planning and care. Parents are likely to feel anxious and guilt on learning of the existence of a congenital anomaly and require sensitive counseling. ${ }^{10}$

The prevalence rate of congenital anomaly is increasing due to exposure of teratogens of various kinds, particularly pesticides but also pharmaceutical products. Congenital anomalies contribute a significant proportion of infant mortality as well as fetal morbidity. As a consequence, it is essential to have basic epidemiological information of these anomalies. Congenital anomaly rates can also used for planning health service. ${ }^{11}$

In the tropics, malnutrition and infections are main causes of infant morbidity and mortality while in the temperate zones, cancer, accidents and congenital abnormalities are the key causes of infant morbidity and mortality.

No national survey or hospital based statistics regarding congenital anomalies in Bangladesh is available till date. But a high number of congenital abnormal babies are delivered at BSMMU (a tertiary referral hospital), Bangladesh each year. So a study has been undertaken to serve as a reference point for actual picture of congenital anomalies in this tertiary care centre and it will also generate data of congenital anomalous fetuses that will help national registry in future. Structural anomalies are considered overt when they are visible on inspection, otherwise they are considered "occult". Considering the elimination or control of some infectious diseases, congenital anomalies are increasingly playing a major role in the mortality and morbidity of children. Congenital anomalies are a major cause of admission and prolonged stay in nursery; they are also an important cause of early and late neonatal deaths. The causes for these anomalies are multifactorial. ${ }^{12}$ 
Prevalent studies of congenital anomalies are useful to establish baseline rates, to document changes over time and to identify clues to aetiology. They are also important for health services planning and evaluating antenatal screening in populations with high risk. The study is also important as it may help to raise the awareness of surgical pediatric intervention and to emphasize the loss of babies with congenital abnormalities. ${ }^{13}$

Steps one can take throughout pregnancy to avoid environmental effects on congenital anomalies include-stop smoking, eating a healthy diet, maintaining a healthy weight, medical management of pre-existing conditions, taking folic acid, avoiding exposure to alcohol and drugs during pregnancy, avoiding exposure to harmful substances, lowering risk for infection, taking daily vitamins, dealing with domestic violence etc. ${ }^{14}$

Most birth defects cannot be cured; treatment focuses on managing the symptoms. In some cases, however, there are ways to treat specific birth defects which may be gene therapy, enzyme replacement therapy, surgery or prenatal treatment.

\section{MATERIALS AND METHODS}

Methodology. The aims and objectives of this study were to determine the prevalence of congenital abnormalities among patients; to describe the different forms of abnormalities, to determine the various birth and maternal characteristics and to see the ultimate outcome of congenital abnormality. For this study purpose congenital abnormalities are defined as obvious abnormality of structure or form which is present at birth or noticed within a few days after birth.

A cross-sectional retrospective study was conducted in which a review of the records of all congenital anomalous patients admitted in different hospitals under Sylhet division in Bangladesh over a four month period (October 2012 to January 2013) was undertaken. Study population consisted of sixty ladies (pregnant or mother) with their anomalous babies. The folders (case files) of these babies were retrieved from the hospital records department and examined individually by the investigators. Data collection was done with structured forms designed for the study. The diagnosis of congenital abnormality was based on clinical evaluation and ultrasound examination (as documented by doctors in the patients' folders). Patient's history, including antenatal history, history of exposure to teratogens and family history of consanguinity were obtained from these folders. Further information obtained includes maternal age, type of delivery, gestational age and type of congenital abnormality. Significant maternal illness like diabetes mellitus, hypertension, hypothyroidism, infection with rubella, toxoplasmosis, herpes simplex, HIV, syphilis, and also exposure to radiation and smoking during antenatal period were included. The prevalence rate was estimated as a per cent of the total number of babies admitted in the unit within the period of the study (number of babies with congenital abnormalities/total number of babies admitted in the hospital for the duration of study).

Statistical methods. Data was analyzed using SPSS 13. Rates and proportions were calculated with 95\% confidence intervals. The proportions were compared using students T-test. Level of significance was set at $\mathrm{P}<0.05$. The sample size of 60 subjects was determined to provide $80 \%$ statistical power and to detect 0.15 differences of expected proportions between two treatment groups of patients at a significance level of 0.05 .

\section{RESULTS AND DISCUSSION}

During the 4 months of the study period sixty patients were noted to have congenital anomalies. Most of anomalous babies' age ranges from zero to bellow 15 years and was frequently detected at the age range of zero to less than 5 years (43\%) (Table 1 ). The chances of detecting abnormality declines with age of the baby e.g. at the age of above 15, possibility of the abnormality is $13.72 \%$ (Table 1 ). Among them $45 \%$ babies were male and $55 \%$ were female $(\mathrm{N}=60)$ (Table 2). Upon analysis of the obstetric history it was found that about $88.33 \%$ of mothers have no history of congenital abnormality 
(Table 3) and about $41.67 \%$ child birth was through cesarean section (Table 4). The most frequently occurring anomaly was associated with Central Nervous System (28.33\%) which was followed by anomalies of the heart. Cases of congenital heart disease constituted $15 \%$ of the total anomalies (Table 5). Gastrointestinal anomalies of which imperforate anus was the most commoncontributes $22.73 \%$ (Table 6). About $6.67 \%$ percent was presented with musculoskeletal anomalies out of which patau syndrome was the most common (18.18\%) (Table 6). Most of the admitted patients (40\%) were discharged after necessary investigations and counselling, 25\% expired, $20 \%$ were referred to other hospitals for further management, mostly including cardiac cases or surgical cases.

Table 1. Distribution of child birth characteristics.

\begin{tabular}{cc}
\hline Characterstics & $\%$ \\
\hline \multicolumn{2}{c}{ Gender } \\
Male & 45.00 \\
Female & 55.00 \\
Birth weight $[\mathrm{Kg}]$ \\
$<2.5$ & 38.33 \\
$2.6-4$ & 31.67 \\
$>4$ & 30.00 \\
Length $[\mathrm{cm}]$ & \\
$<50$ & 43.33 \\
$>50$ & 56.67 \\
\hline
\end{tabular}

Table 2. Age distribution of patients.

\begin{tabular}{cc}
\hline Age group (yrs) & Percentage (\%) \\
\hline 1-less than 5 & $43 \%$ \\
6-less than 10 & $29 \%$ \\
$10-15$ & $14.28 \%$ \\
Above 15 & $13.72 \%$ \\
\hline
\end{tabular}

All expired patients were in the early neonatal or perinatal age. Of these, $15 \%$ patients left without medical advice. In $68 \%$ of patients with complications like prematurity, low birth weight, respiratory distress, sepsis, intestinal obstruction and seizures etc. were present. Thirty-two percent patients presented with multiple anomalies for example patient with Down's syndrome also presented with dextrocardia or congenital heart defects, based upon the obvious defects such cases were included in only one group to avoid repetition. Cross-tabulation was used to correlate variables of gender, type of defects and outcome of the patients (Table 4).

Table 3. Description of the congenital abnormalities and their outcome.

\begin{tabular}{lc}
\hline Congenital abnormality observed & $\%$ \\
\hline Ano-rectal Malformation [Imperforate & 22.73 \\
Anus] & 13.64 \\
Left Cleft-lip/Palate & \\
Multiple Congenital Anomaly [Patau & 18.18 \\
Syndrome] & 9.09 \\
Myelomeningocele [Ruptured] & 4.55 \\
Myelomeningocele/Hydrocephalus/Bilateral & 9.09 \\
Occipital Cranial Bifidum/Ruptured & 9.09 \\
Occipital Encephalocele & 13.63 \\
Omphalocele minor & \\
\hline
\end{tabular}

Table 4. Maternal birth characteristics.

\begin{tabular}{cc}
\hline Characteristic & $\%$ \\
\hline Maternal age [yrs] & \\
$20-25$ & 28.33 \\
$26-30$ & 35 \\
$31-35$ & 23.34 \\
$36-40$ & 13.33 \\
Mode of delivery & \\
Spontaneous vertex & 58.33 \\
Caesarean section & 41.67 \\
\hline
\end{tabular}

This study was an effort to find out an actual picture of congenital anomalous babies born in these different hospitals. The prevalence rate of $2.8 \%$ obtained in this study does not reflect the picture in the general population as this was purely a hospital based study with no attempt whatsoever to obtain a sample that would be representative of the general population. Be that as it may, it is possible that a community based study or one taking into account all deliveries occurring in the larger society may yield a higher prevalence. In our part of the work, for instance, some babies with congenital abnormalities 
brought to teaching or specialist hospitals do not present to the neonatology unit but are seen at other specialist units such as paediatric surgery unit or neuro-surgery unit etc. and a study conducted at the neonatology unit per se as is the case in this work may not be able to "capture" these other babies. Some that are born outside the hospital with congenital abnormalities are not taken to hospitals for care but are taken to traditional healers or other alternative practitioners while some are just left at home to their fate. Some that are brought to peripheral hospitals may not be referred to tertiary or teaching hospitals for care. Needless to mention that majority of these babies are not well managed and a good number die or are left with avoidable complications.

All the mothers whose babies had congenital abnormalities booked for antenatal care in the second trimester or later. Ignorance and poverty may have accounted for this. In north-east Nigeria noted that about $90 \%$ of women who had children with birth defects did not attend ante-natal clinic but preferred to deliver their babies outside the teaching hospital with traditional birth attendants in attendance.

Brain abnormalities (meningocoele, myelocoele and encaphalocoele) are observed to occur more frequently than other abnormalities in this study. The reasons adduced above, especially lack of folic acid supplementation, may explain the increased occurrence of these disorders in our series. However the reason for this predominance could also be less related to folic acid deficiency than to the fact that these congenital abnormalities are severe enough to require quick intervention but usually survive for surgery. Another possible explanation for this is that these disorders may be perceived by the parents as being severe, with an increased likelihood of their seeking medical support compared to other abnormalities such as musculoskeletal abnormalities which may be perceived as being less severe.

Limitations. A retrospective, cross-sectional study of this nature is bound to face a number of challenges, and expectedly so, as the investigators are not fully "in-charge" of the processes. Firstly, retrieving patients' folders from the hospital record department (which is yet to be fully computerized) was a rather difficult task; some of the folders retrieved contained inadequate information and this affected the quality of the study. In addition, a hospital based study of this nature, especially one restricted to only a section of the hospital as is the case in this instance, cannot be said to reflect truly what could be obtained in general population. A prospective, community based study is thus desirable.

\section{CONCLUSION}

Congenital anomalies make an important contribution to infant mortality. They remain a leading cause of death in many countries in the world. Mortality of infants born with congenital anomalies varies with the type of anomaly, being highest among those with central nervous system, cardiovascular system, respiratory and genetic disorders. Screening of high risk cases, routine prenatal folic acid supplementation, early prenatal diagnosis and termination of fetus with lethal anomaly before attaining viability will reduce perinatal morbidity and mortality. More stress needs to be laid on prescribing pre-conception vitamin supplementations. Extensive collaboration between the obstetrician and pediatricians is required for antenatal diagnosis of many congenital anomalies so that proper parental education and counseling may be done. To draw significant conclusions it is recommended that all neonates should be examined with scrutiny for overt as well as occult congenital anomalies. Moreover, it is necessary to establish a registry system for congenital anomalies.

\section{REFERENCES}

1. Ambe, J.P., Nadziga, A.G., Akpede, G.O. and Mava, Y. 2010. Pattern and outcome of congenital abnormality in newborn babies in a Nigerian Teaching Hospital. West. Afr. Med. 29, 24-29.

2. Sawardeker, K.P. 2005. Prevalence of isolated minor congenital abnormalities in a regional hospital in Oman. Saudi. Med. J. 26, 1567-1572. 
3. Bahauddin, I.S., Hosan, M.S., Attyyaa, R.A. and Suleiman, A. 2008. Antenatal diagnosis and prevalence and outcome of major congenital abnormality in Saudi Arabia; a hospital based study. Ann. Saudi. Med. J. 28, 272-276.

4. Kumar, V. 2010. Congenital abnormality of the brain. (In Robbins and Cotran's Pathological Basis of Diseases 7th edition. (Edited by Kumar, V., Abbas, A. and Fausto, N.) WB Saunders, Seattle, 470.

5. Chaturvedi, P. and Banerjee, K.S. 1989. Spectrum of congenital malformations in the newborns from rural Maharash. The. Ind. J. Ped. 56, 501-507.

6. Kalter, H. and Warkany, J. 1983. Medical progress. Congenital malformations: Etiologic factors and their role in prevention. N. Eng. J. Med. 308, 424-433.

7. Jensen, T.K. 2002. Congenital malformations, in: Children's health and environment: A review of evidence. A joint report from the European Environment Agency and the WHO Regional Office for Europe. Office for Official Publications of the European Communities. pp. 116-126.

8. Fouzia, P. and Subhana, T. 2007. Frequency and pattern of distribution of congenital anomalies in the newborn and associated maternal risk factor. J. Coll. Phy. Surg. Pak. 17, $340-343$.
9. Agha, M.M., William, J.I., Marrett, L. and Dodds, L. 2006. Detrimants of survival in children with severe congenital anomalies: A long term population based cohort study. Paediatrics 76, 46-54.

10. Wynshaw-Boris, A. and Biesecker, L.G. 2007. (Dysmorphology: In: Kliegman, R.M., and Behrman, R.E. Editors.) Nelson text book of Pediatrics.18th Edition Philadelphia: Saunders, pp. 786-787.

11. Lee, K., Khoshnood, B., Chen, L., Wall, S., Cromie, W. and Mittendorf, R. 2000. Infant Mortality from Congenital Malformations in the United States, 1990-1997, Obs. Gynae. 98, 620-627.

12. Mosayebi, Z. and Movahedian, A.H. 2007. Pattern of congenital malformations in cosanguineous versus non consanguineous marriages in Kashan, Islamic republic of Iran. Health. J. 13, 868-875.

13. Muga, R.O., Mumah, S.C.J. and Juma, P.A. 2009. Congenital malformations among newborns in Kenya. Afr. J. Food. Agri. Nutr. Dev. 19, 819-829.

14. Maitra, A. and Kumar, V. 2003. Genetic and Pediatric Diseases (Cotran, R.S., Collins, T. and Robbins, S.L. (Eds.). Robbin's Basic Pathology). Philadelphia: W.B. Saunders, pp. 211-263. 